\title{
Ajuste y predicción de la distribución Weibull a las estructuras diamétricas de plantaciones de pino de Durango, México
}

\author{
David Maldonado Ayala ${ }^{1}$ \\ José Návar Chaidez ${ }^{2}$
}

\begin{abstract}
RESUMEN
En este trabajo se ajustaron y predijeron los parámetros de la distribución Weibull con atributos del rodal. Se demostró mediante pruebas de hipótesis que las ecuaciones son eficientes para predecir las estructuras diamétricas de los rodales utilizados en el ajuste y de rodales seleccionados para pruebas de validación. Por esta razón se recomiendan las ecuaciones en la predicción de las estructuras diamétricas de las plantaciones presentes en el estado de Durango, México (Pinus durangensis, $P$. cooperi, $P$. engelmannii, $P$. Cooperi, y $P$. arizonica)..
\end{abstract}

PALABRAS CLAVE:

Estimación de parámetros, momentos, prueba de hipótesis, tres parámetros.

\begin{abstract}
In this research the parameters of the Weibull distribution were estimated and predicted with stand attributes. Using hypothesis testing procedures, it was demonstrated that the equations to predict parameters, efficiently mimicked the diameter structures of forest stands used in the fitting as well as forest stands used in validating the prediction equations. Therefore, the equations are recommended to estimate the diameter structures of forest plantations of Durango, Mexico (Pinus durangensis, $P$. cooperi, $P$. engelmannii y $P$. arizonica).
\end{abstract}

KEY WORDS:

Moments, parameter estimation, testing hypothesis, three parameters. 


\section{INTRODUCCIÓN}

El manejo de los recursos maderables requiere de la modelación probabilística de las estructuras diamétricas. Éstas se han predicho por una diversidad de funciones probabilísticas, dentro de las que destacan la Gamma, Lognormal, Beta, Johnson SB, Charlier y Weibull (Návar y Corral, 2000; Návar y Contreras, 2000; Tang y Pearson, 1992; Gupta et al., 1992; Laar et al., 1989; Jayaraman y Rugmini, 1988; Li, 1987; Svalov, 1984; Gadow, 1984; Kou, 1982; Hafley y Schreuder, 1977; Seth et al., 1975; Bailey y Dell, 1973). La distribución Weibull, introducida por el físico sueco Waloddi Weibull (Devore, 1987), fue propuesta para múltiples aplicaciones y es la herramienta clásica en la estimación del crecimiento y productividad forestal con la predicción de estructuras diamétricas (Vanclay, 1994), por su forma cerrada y su flexibilidad en simular diferentes formas, escalas o posiciones diamétricas. Su desventaja principal es su unimodalidad. Clutter et al. (1983) y Wenger (1984) describen ejemplos de su aplicación en el manejo forestal.

Návar et al. (2002); Návar y Corral (2000); Návar y Contreras (2000); Evans et al. (1989); Laar et al. (1989); Shiver (1998); Ueno y Osawa (1987); Zarnoch y Dell (1985); Zutter et al. (1982) y Pierce (1976) compararon diferentes procedimientos de ajuste de parámetros, dentro de los cuales destacan: máxima verosimilitud de 2 y 3 parámetros, porcentajes o ajuste de puntos y momentos. El desarrollo de modelos de rendimiento e incremento al nivel del rodal, requieren de distribuciones probabilísticas probadas por medio de estas comparaciones, las cuales no se han realizado para describir las estructuras diamétricas de rodales plantados con especies de Pinus de la Sierra Madre Occidental del estado de Durango, México.

Návar et al. (2002) ajustaron los parámetros de la distribución Weibull y los modelaron por diferentes técnicas para bosques naturales. Torres-Rojo et al. (1992) realizaron investigaciones similares en plantaciones de Pinus patula. Este tipo de trabajos debe ser realizado para poder tomar decisiones sobre las plantaciones forestales del estado de Durango, México.

\section{OBJETIVOS}

1) Estimar parámetros de la distribución Weibull, 2) predecir parámetros de la misma distribución y 3) validar el ajuste de la predicción de parámetros de distribución Weibull obtenidos con el método de momentos, a rodales plantados con cuatro especies de pino (Pinus durangensis, $P$. cooperi, $P$. engelmannii y $P$. arizonica) de Durango, México.

\section{ÁREA DE ESTUDIO}

Los estudios para ajustar parámetros, predecirlos y validarlos se realizaron en plantaciones forestales de la Unidad de Conservación y Desarrollo Forestal No 6. de El Salto, Durango, México. El área donde se plantaron las especies de pino se caracteriza por ser la parte alta del macizo principal de la Sierra Madre Occidental del centro-sur de Durango. El clima del área es templado frío con lluvias veraniegas, con una temperatura y precipitación promedio anual de $11.7^{\circ} \mathrm{C}$ y $1200 \mathrm{~mm}$, respectivamente. Los suelos son predominantemente Litosoles, Rendzinas y Cambisoles, someros, con textura media, generalmente francos. La vegetación previa a disturbios naturales y antropogénicos estuvo caracterizada por bosques mixtos de coníferas y latifoliadas, con abundancias relativas caracterizadas por la altitud sobre el nivel del mar y la pendiente (Graciano, 2001). Las especies de pino que dominaron el paisaje fueron: Pinus durangensis, $P$. cooperi, $P$. teocote, $P$. leiophylla y $P$. engelmannii. Las especies de encino características son: Quercus rubra, $Q$. virginiana, $Q$. potosina, 
Q. nigra, $Q$. sideroxyla, $Q$. cupreata y $Q$. crassifolia.

Las plantaciones se realizaron en sitios perturbados por incendios preferencialmente y se realizaron en parcelas de diferentes dimensiones y espaciamientos, dominando los de $2 \times 2 \mathrm{~m}$.

\section{METODOLOGÍA}

La información utilizada se colectó a través del establecimiento de sitios temporales de muestreo (parcelas), de forma cuadrada, generalmente de $600 \mathrm{~m}^{2}$ de superficie, de los cuales, 23 se seleccionaron aleatoriamente de entre 9 rodales. Los árboles fueron medidos en su diámetro normal, altura total, cobertura y edad. La Tabla 1 presenta los valores estadísticos de los árboles medidos por parcela.

\section{La Distribución Weibull}

La distribución probabilística Weibull, como función de densidad probabilística (fdp) está dada por el modelo matemático (1) y como función de densidad acumulada (fda) por el modelo (2) (Haan, 1986).

$$
\begin{gathered}
P_{X}(X)=\alpha(X-\varepsilon)^{\alpha-1}(\beta-\varepsilon)^{-\alpha} e^{\left\{-\left[\frac{X-\varepsilon}{\beta-\varepsilon}\right]^{\alpha}\right\}} \\
P_{X}(X)=1-e^{\left\{-\left[\frac{X-\varepsilon}{\beta-\varepsilon}\right]^{\alpha}\right\}}
\end{gathered}
$$

donde:

$$
\begin{aligned}
P_{x}(x)= & \text { probabilidad de la variable } \\
& \text { aleatoria } x \\
\alpha, \beta \text { y } \varepsilon= & \text { parámetros de forma, escala } y \\
& \text { posición } \\
e \quad & \text { antilogaritmo de la variable } \\
& \text { aleatoria } x
\end{aligned}
$$

Los parámetros $\alpha, \beta$ y $\varepsilon$ fueron ajustados por medio del procedimiento de momentos sin ponderar. Hahn y Shapiro (1967) indican que el coeficiente de asimetría $(\gamma)$ está relacionado con $\alpha$ por:

$$
\gamma=\frac{\Gamma(1+3 / \alpha)-3 \Gamma(1+2 / \alpha) \Gamma(1+1 / \alpha)+2 \Gamma^{3}(1+1 / \alpha)}{\left[\Gamma(1+2 / \alpha)-\Gamma^{2}(1+1 / \alpha)\right]^{3 / 2}}
$$

y con esto definieron:

$$
\begin{aligned}
& \beta=\mu+\sigma A(\alpha) \\
& \varepsilon=\beta-\sigma B(\alpha)
\end{aligned}
$$

donde $\sigma$ es la desviación estándar de la variable aleatoria $x$ y $A(\alpha)$ y $B(\alpha)$ son:

$$
\begin{gathered}
A(\alpha)=[1-\Gamma(1+1 / \alpha) B(\alpha)] \\
B(\alpha)=\left[\Gamma(1+2 / \alpha)-\Gamma^{2}(1+1 / \alpha)\right]^{-1 / 2}
\end{gathered}
$$

\section{Procedimiento}

El ajuste de parámetros se realizó en programas de cómputo elaborados previa-mente por Návar y Contreras (2000) y se basan en ajustar $\alpha$ con $\gamma$ por medio de la ecuación (3), para posteriormente encon-trar $\beta$ y $\varepsilon$ por medio de las ecuaciones (4) y (5) con el auxilio de (6) y (7). Con los estimadores de los parámetros, se calcularon las frecuencias de las clases diamétricas y junto con las frecuencias observadas se aplicaron las pruebas de bondades de ajuste por $\chi^{2} y$ Kolmogorof-Smirnoff (K-S). La primera se trabajó con las frecuencias absolutas para intervalos de clase de $2.5 \mathrm{~cm}$ estimadas con la ecuación (1) y la segunda con las probabilidades diamétricas acumuladas y estimadas con la ecuación (2).

En la predicción de parámetros se utilizaron 18 parcelas seleccionadas aleatoriamente y en la prueba de validación se utilizaron las 5 parcelas restantes. 
Tabla 1. Parámetros dasométricos promedio de especies típicas de plantaciones forestales de la Sierra Madre Occidental de Durango, México

\begin{tabular}{|c|c|c|c|c|c|c|}
\hline \multirow{2}{*}{ PARCELA:ESPECIES } & \multirow{2}{*}{$\mathrm{DB}(\mathrm{cm})$} & \multirow{2}{*}{$\begin{array}{c}\mathrm{H} \\
(\mathrm{m})\end{array}$} & \multirow{2}{*}{$\begin{array}{c}\text { RCOPA } \\
(\mathrm{m})\end{array}$} & \multirow{2}{*}{$\begin{array}{l}\text { EDAD } \\
\text { (años) }\end{array}$} & \multicolumn{2}{|c|}{ DENSIDAD No ha ${ }^{-1}$} \\
\hline & & & & & Inicial & Edad t \\
\hline LE1: Pinus durangensis & 12.24 & 8.15 & 1.0 .3 & 21 & 6667 & 4667 \\
\hline LE2: $P$. arizonica & 10.60 & 5.78 & 1.04 & 21 & 6667 & 4217 \\
\hline LE3: P. cooperi & 15.52 & 7.34 & 1.18 & 21 & 6667 & 1900 \\
\hline LE4: $P$. arizonica & 9.55 & 4.61 & - & 21 & 6667 & 5500 \\
\hline LE5: P. cooperi & 15.69 & 9.77 & - & 21 & 6667 & 2450 \\
\hline LE6: $P$. cooperi & 15.93 & 8.63 & 1.27 & 21 & 6667 & 2882 \\
\hline LE7: P. durangensis & 14.77 & 7.23 & 1.24 & 21 & 6667 & 3138 \\
\hline LE8: P. durangensis & 14.84 & 8.91 & 1.22 & 21 & 6667 & 3090 \\
\hline LE9: P. cooperi & 10.19 & 4.81 & 0.97 & 21 & 6667 & 3100 \\
\hline SA1: P. durangensis & 16.27 & 6.06 & 1.25 & 17 & 2500 & 1867 \\
\hline SA2: $P$. durangensis & 20.31 & 7.20 & 0.90 & 17 & 1111 & 689 \\
\hline SA3: P. durangensis & 17.56 & 6.41 & 1.45 & 17 & 1600 & 1444 \\
\hline SA4: P. durangensis & 14.43 & 6.14 & 1.94 & 17 & 4444 & 2633 \\
\hline PI1: P. cooperi B & 14.00 & 3.63 & - & 18 & 2500 & 1633 \\
\hline AL1: P. durangensis & 15.68 & 7.30 & 1.09 & 16 & 5000 & 2233 \\
\hline AL2: P. cooperi & 11.03 & 5.83 & 1.65 & 16 & 5000 & 1817 \\
\hline SP1: $P$. engelmannii & 11.16 & 2.87 & 1.06 & 10 & 2500 & 1617 \\
\hline SP2: P. cooperi & 8.69 & 2.66 & 1.21 & 10 & 2500 & 1867 \\
\hline SP3: $P$. durangensis & 8.41 & 2.81 & 1.38 & 10 & 2500 & 2133 \\
\hline LB1: P. engelmannii & 5.61 & 2.80 & 0.59 & 11 & 10000 & 3575 \\
\hline LB2: P. cooperi & 10.55 & 5.84 & 1.11 & 11 & 10000 & 4950 \\
\hline LC1: P. durangensis & 6.32 & 2.26 & 1.29 & 7 & 2500 & 1767 \\
\hline LC2: P. cooperi & 4.97 & 1.74 & 1.10 & 7 & 2500 & 1950 \\
\hline $\begin{array}{l}\text { DB = Diámetro basal }(\mathrm{cm}) \\
\mathrm{H}=\text { altura }(\mathrm{m})\end{array}$ & $\begin{array}{l}\text { Rcopa } \\
\text { Edad t }\end{array}$ & $\begin{array}{l}\text { radio } \\
\text { Edac }\end{array}$ & a planta & al mome & de la $\mathrm{m}$ & ón (años) \\
\hline
\end{tabular}


Las ecuaciones de predicción de parámetros se generaron con los atributos dasométricos del rodal, diámetro promedio, diámetro cuadrático promedio, altura dominante, altura promedio, área basal, cobertura promedio por medio de regresión lineal y no lineal múltiple, usando el procedimiento paso a paso (stepwise) en SAS.

\section{RESULTADOS Y DISCUSIÓN}

\section{Estimación de parámetros}

Los parámetros estimados y predichos por las ecuaciones de predicción se reportan en la Tabla 2. Los parámetros son similares debido a que las ecuaciones de predicción generaron estadísticos confiables. Éstos se presentan en la Tabla 3.

\section{Pruebas de Bondad de Ajuste}

Las hipótesis nulas y alternas para los parámetros estimados y predichos por las ecuaciones se presentan en la Tabla 4. En esta tabla se destaca que la hipótesis nula $\left(\mathrm{H}_{0}\right)$, con un error $=0.05 \mathrm{se}$ aceptó en 11 de 18 veces con la prueba de bondad de ajuste de $\chi^{2}$. Con la prueba de K-S, el número de hipótesis nulas incrementa hasta el $100 \%$, es decir, la distribución Weibull se ajusta adecuadamente a los 18 rodales utilizados en la estimación de parámetros.

Tabla 2. Parámetros estimados y predichos de la distribución probabilística Weibull ajustada a los diámetros de 18 rodales plantados con 4 especies de pinos en

Durango, México

\begin{tabular}{|c|c|c|c|c|c|c|c|}
\hline \multirow[t]{2}{*}{ RODAL } & \multirow[t]{2}{*}{ ESPECIE } & \multicolumn{3}{|c|}{$\begin{array}{l}\text { PARÁMETROS DE LA } \\
\text { DISTRIBUCIÓN WEIBULL }\end{array}$} & \multicolumn{3}{|c|}{$\begin{array}{l}\text { PARÁMETROS PREDICHOS DE } \\
\text { LA DISTRIBUCIÓN WEIBULL }\end{array}$} \\
\hline & & $\alpha$ & $\beta$ & $\varepsilon$ & $\alpha$ & $\beta$ & $\varepsilon$ \\
\hline 1 & Pinus durangensis & 2.977 & 13.486 & 1.873 & 2.825 & 13.570 & 1.645 \\
\hline 2 & P. durangensis & 4.197 & 16.499 & -1.708 & 2.417 & 16.270 & 3.027 \\
\hline 3 & P. durangensis & 2.485 & 17.620 & 5.639 & 2.131 & 17.590 & 4.993 \\
\hline 4 & $P$. durangensis & 3.618 & 19.172 & 2.723 & 2.092 & 18.849 & 6.615 \\
\hline 5 & P. durangensis & 3.423 & 15.549 & 4.454 & 2.952 & 15.640 & 3.432 \\
\hline 6 & P. durangensis & 3.641 & 17.087 & 2.814 & 2.600 & 17.017 & 3.907 \\
\hline 7 & P. durangensis & 2.733 & 7.058 & 0.391 & 2.046 & 6.997 & 0.576 \\
\hline 8 & P. cooperi & 3.257 & 16.914 & 5.103 & 3.541 & 17.212 & 2.304 \\
\hline 9 & P. cooperi & 2.669 & 17.365 & 4.417 & 2.465 & 17.426 & 3.442 \\
\hline 10 & P. cooperi & 2.222 & 11.319 & 1.431 & 1.552 & 11.294 & 1.618 \\
\hline 11 & P. cooperi & 2.907 & 12.374 & -0.083 & 1.729 & 12.157 & 1.658 \\
\hline 12 & $P$. engelmannii & 4.852 & 13.519 & -9.959 & 0.890 & 13.944 & 5.328 \\
\hline 13 & P. cooperi & 3.658 & 11.872 & -1.572 & 2.062 & 11.639 & 1.950 \\
\hline 14 & P. cooperi & 1.905 & 9.092 & 5.570 & 3.501 & 9.421 & 1.292 \\
\hline 15 & P. cooperi & 3.832 & 5.574 & -0.689 & 2.900 & 5.520 & 0.296 \\
\hline 16 & $P$. arizonica & 2.688 & 10.333 & 3.278 & 3.200 & 10.493 & 1.477 \\
\hline 17 & $P$. engelmannii & 3.798 & 12.078 & 2.536 & 2.554 & 11.986 & 2.940 \\
\hline 18 & P. cooperi & 3.946 & 15.158 & 2.902 & 2.340 & 14.973 & 5.205 \\
\hline
\end{tabular}


Tabla 3. Ecuaciones para predecir parámetros de la distribución probabilística Weibull ajustada a los diámetros de 18 rodales plantados con 4 especies de pinos en

Durango, México

\begin{tabular}{|c|c|c|c|c|}
\hline \multirow{2}{*}{\multicolumn{2}{|c|}{ ECUACIÓN }} & \multicolumn{3}{|c|}{$\begin{array}{l}\text { ESTADÍSTICOS DE } \\
\text { AJUSTE }\end{array}$} \\
\hline & & $\mathrm{R}^{2}$ & Sx & Sx(\%) \\
\hline$\beta$ & $=\mathrm{e}(0.1567+0.946 * \ln (\mathrm{D})+0.033375 * \ln (\mathrm{Ho}))$ & 0.99 & 0.22 & 1.6 \\
\hline$\alpha$ & $=\mathrm{e}\left(-0.198+21.0157^{*} \ln (\beta)-20.89 \ln (\mathrm{Dq})-0.1364 \ln (\mathrm{H})\right)$ & 0.92 & 1.59 & 48.7 \\
\hline$\varepsilon$ & $\begin{aligned}= & e(0.911-44.66 \ln (\beta)+31.485 \ln (D)+14.03 \ln (D q)+ \\
& 0.351099 \ln (D))\end{aligned}$ & 0.97 & 3.87 & 46.3 \\
\hline $\begin{array}{l}\mathrm{D} \\
\mathrm{H} \\
\mathrm{Dc}\end{array}$ & $\begin{array}{llr}=\text { Diámetro basal }(\mathrm{cm}) & \mathrm{Ho}=\text { Altura dominante }(\mathrm{m}) \\
=\text { Altura total }(\mathrm{m}) & \alpha, \beta, \varepsilon=\text { Parámetros de forma, es } \\
=\text { Diámetro cuadrático basal }(\mathrm{cm}) & & \text { Weibull, respectivamente }\end{array}$ & & & \\
\hline
\end{tabular}

Tabla 4. Las pruebas de bondad de ajuste de $\chi^{2}$ y de KS de la distribución Weibull ajustada a los diámetros de 18 rodales plantados en Durango, México

\begin{tabular}{|c|c|c|c|c|c|c|c|}
\hline \multirow[b]{2}{*}{ RODAL } & \multirow[b]{2}{*}{ ESPECIE } & \multicolumn{3}{|c|}{ PRUEBA DE $\chi^{2}$} & \multicolumn{3}{|c|}{ PRUEBA DE K-S } \\
\hline & & $\begin{array}{l}\text { Valor } \\
\text { de } \chi^{2}\end{array}$ & $\begin{array}{l}\text { Prob. } \\
\text { de } \chi^{2}\end{array}$ & Hipótesis & $\begin{array}{l}\text { Valor } \\
\text { de KS }\end{array}$ & $\begin{array}{c}\text { Valor } \\
\text { tabular } \\
\text { de KS }\end{array}$ & Hipótesis \\
\hline 1 & Pinus durangensis & 12.711 & 0.0479 & $\mathrm{Ha}$ & 0.0813 & 0.0375 & Ho \\
\hline 2 & P. durangensis & 7.952 & 0.3368 & $\mathrm{Ho}$ & 0.1442 & 0.0776 & Ho \\
\hline 3 & P. durangensis & 5.015 & 0.5419 & Ho & 0.1285 & 0.0939 & Ho \\
\hline 4 & P. durangensis & 35.664 & 0.0000 & $\mathrm{Ha}$ & 0.1687 & 0.1192 & Ho \\
\hline 5 & P. durangensis & 9.951 & 0.0413 & $\mathrm{Ha}$ & 0.1530 & 0.0608 & Ho \\
\hline 6 & P. durangensis & 9.492 & 0.2193 & Ho & 0.1175 & 0.0538 & Ho \\
\hline 7 & P. durangensis & 0.896 & 0.6387 & Ho & 0.1321 & 0.0565 & Ho \\
\hline 8 & P. cooperi & 4.130 & 0.5308 & Ho & 0.1374 & 0.0651 & Ho \\
\hline 9 & P. cooperi & 14.501 & 0.0430 & $\mathrm{Ha}$ & 0.1122 & 0.0608 & Ho \\
\hline 10 & P. cooperi & 15.855 & 0.0073 & $\mathrm{Ha}$ & 0.0997 & 0.0693 & Ho \\
\hline 11 & P. cooperi & 11.007 & 0.0882 & Ho & 0.1303 & 0.0383 & Ho \\
\hline 12 & P. engelmannii & 18.072 & 0.0117 & $\mathrm{Ha}$ & 0.1137 & 0.0738 & Ho \\
\hline 13 & P. cooperi & 2.755 & 0.7377 & Ho & 0.0789 & 0.0256 & Ho \\
\hline 14 & P. cooperi & 8.939 & 0.0115 & $\mathrm{Ha}$ & 0.1285 & 0.0973 & Ho \\
\hline 15 & P. cooperi & 2.575 & 0.2759 & Ho & 0.1257 & 0.0333 & Ho \\
\hline 16 & $P$. arizonica & 2.921 & 0.2321 & $\mathrm{Ho}$ & 0.1834 & 0.0955 & Ho \\
\hline 17 & P. engelmannii & 6.249 & 0.1001 & $\mathrm{Ho}$ & 0.1381 & 0.0817 & Ho \\
\hline 18 & P. cooperi & 6.965 & 0.0730 & Ho & 0.1374 & 0.0641 & Ho \\
\hline
\end{tabular}


Para los rodales utilizados en la validación de los modelos, se destaca que el número de hipótesis nulas se aceptó con un error al 0.05 en 4 de 5 casos para la prueba de $\chi^{2}$ (Fig. 1 y 2). Para la prueba de K-S, se destaca que el $100 \%$ de las hipótesis son nulas, con un error del 0.05. Cuando se utilizan los parámetros predichos por las ecuaciones de la Tabla 3, el número de hipótesis nulas se reduce hasta en 3 de 5 para la prueba de $\chi^{2}$ y en 2 de 5 casos para la prueba de K-S, con un error del 0.05 (Tabla 5).

En otras palabras, el método de momentos para estimar parámetros es adecuado y las ecuaciones de predicción de parámetros también son precisas a pesar del bajo número de rodales utilizados en las pruebas de ajuste y validación.
Existen en la literatura diferentes métodos de estimación de parámetros, los que han sido probados en diferentes ecosistemas. La utilización del método de momentos en la estimación de parámetros es una excepción a la regla. El método de máxima verosimilitud de dos parámetros ha sido convencionalmente utilizado para modelar las estructuras diamétricas de Quercus robur (Laar et al., 1989) y de Pinus elliottii (Shiver, 1998) y de bosques mixtos e irregulares de Durango (Návar et al., 2002; Návar y Corral, 2000; Návar y Contreras, 2000) y es recomendado por estimar parámetros sin sesgo, eficientes, consistentes, con menor varianza y, por requerir un número de datos menor para obtener la solución correcta (Haan, 1986; Ayers y Jackson, 1989; Kilkki et al., 1989; Hokka et al., 1991; Liu et al., 1992).

Tabla 5. Las pruebas de bondad de ajuste y validación de $\chi^{2}$ y de K-S de la distribución probabilística Weibull ajustada a los diámetros de 5 rodales plantados con 4 especies en Durango, México y utilizados en pruebas de validación de modelos

1. Ajuste

\begin{tabular}{llllllll}
\hline RODAL & ESPECIE & \multicolumn{3}{c}{ PRUEBA DE $\chi^{2}$} & \multicolumn{3}{c}{ PRUEBA DE K-S } \\
\cline { 3 - 7 } & & $\begin{array}{c}\text { Valor } \\
\text { de } \chi^{2}\end{array}$ & $\begin{array}{c}\text { Prob. } \\
\text { de } \chi^{2}\end{array}$ & Hipótesis & $\begin{array}{c}\text { Valor tabular } \\
\text { de KS }\end{array}$ & $\begin{array}{c}\text { Valor de } \\
\text { KS }\end{array}$ & Hipótesis \\
\hline 1 & P.durangensis & 4.005 & 0.67597 & Ho & 0.16492 & 0.080742 & Ho \\
\hline 2 & P.durangensis & 5.151 & 0.6416 & Ho & 0.17272 & 0.064371 & Ho \\
\hline 3 & P.durangensis & 5.869 & 0.05317 & Ho & 0.12021 & 0.071194 & Ho \\
\hline 4 & P.cooperi & 8.468 & 0.13226 & Ho & 0.12738 & 0.075055 & Ho \\
\hline 5 & P.arizonica & 16.728 & 0.03307 & Ha & 0.08567 & 0.060169 & Ho \\
\hline
\end{tabular}

2. Validación

\begin{tabular}{|c|c|c|c|c|c|c|c|}
\hline \multirow[t]{2}{*}{ RODAL } & \multirow[t]{2}{*}{ ESPECIE } & \multicolumn{3}{|c|}{ PRUEBA DE $\chi^{2}$} & \multicolumn{3}{|c|}{ PRUEBA DE K-S } \\
\hline & & $\begin{array}{l}\text { Valor de } \\
\chi^{2}\end{array}$ & $\begin{array}{c}\text { Prob de } \\
\chi^{2}\end{array}$ & Hipótesis & $\begin{array}{c}\text { Valor tabular } \\
\text { de KS }\end{array}$ & $\begin{array}{c}\text { Valor de } \\
\text { KS }\end{array}$ & Hipótesis \\
\hline 1 & P.durangensis & 9.004 & 0.1734 & Ho & 0.16492 & 0.12221 & Ho \\
\hline 2 & P.durangensis & 14.799 & 0.0386 & $\mathrm{Ha}$ & 0.17272 & 0.13172 & Ho \\
\hline 3 & P.durangensis & 7.295 & 0.0603 & $\mathrm{Ho}$ & 0.12021 & 0.10862 & Ho \\
\hline 4 & P.cooperi & 40.184 & 0.0000 & $\mathrm{Ha}$ & 0.12738 & 0.19181 & $\mathrm{Ha}$ \\
\hline 5 & P.arizonica & 39.951 & 0.0000 & $\mathrm{Ha}$ & 0.08567 & 0.12558 & $\mathrm{Ha}$ \\
\hline
\end{tabular}



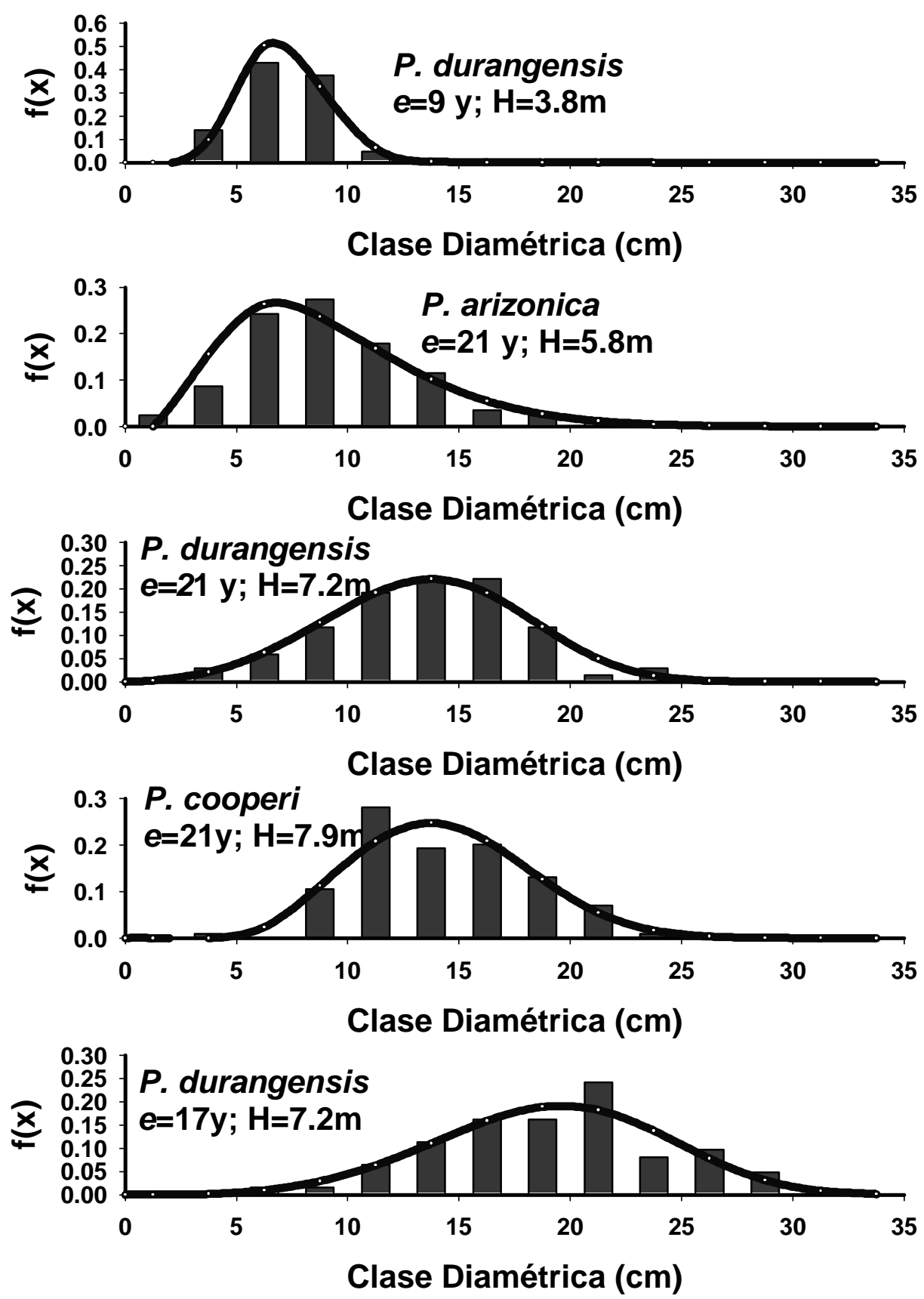

Figura 1. Pruebas de validación de las ecuaciones de predicción de parámetros a través de la prueba de $\chi^{2}$ para cinco parcelas plantadas en Durango, México. 


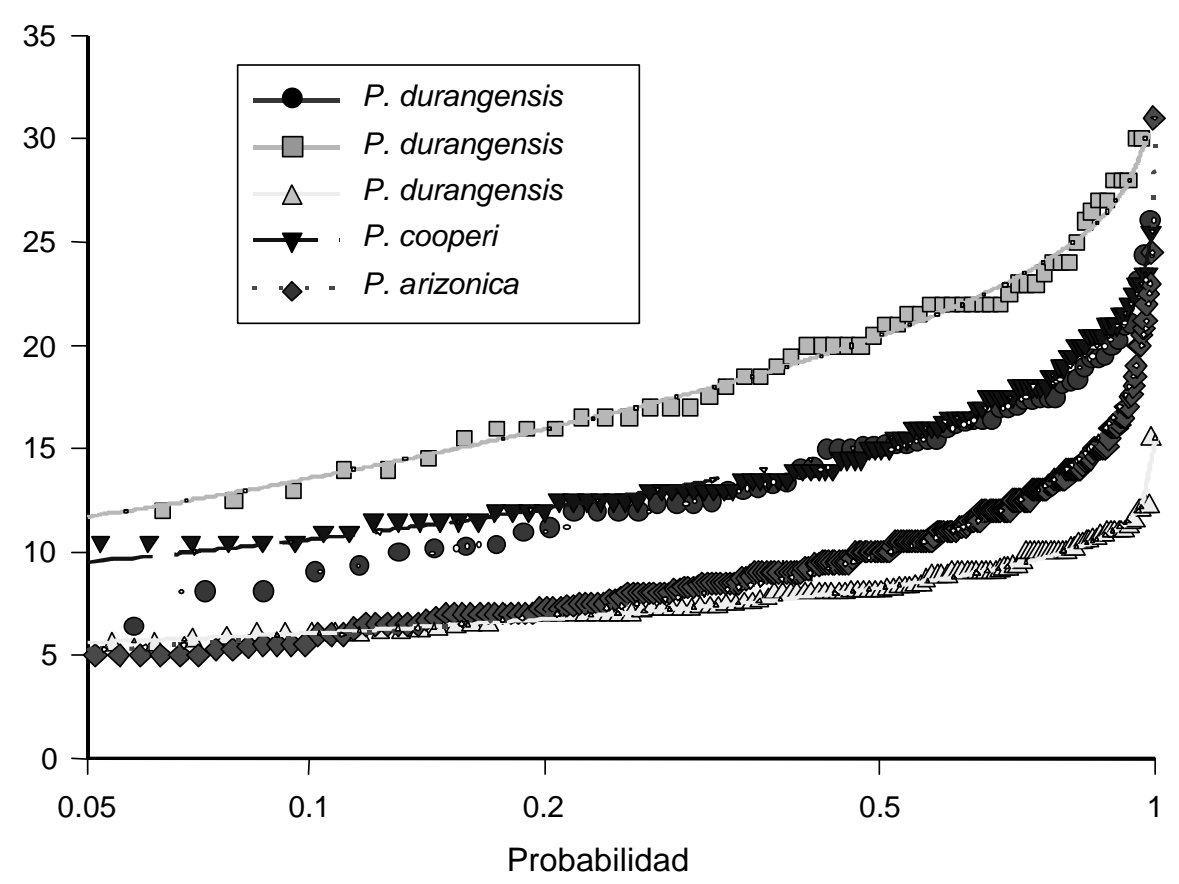

Figura 2. Pruebas de validación de las ecuaciones de predicción de parámetros de la distrubución Weibull para cinco parcelas plantadas en Durango, México.

Sin embargo, la facilidad de estimar parámetros demuestra que el método de momentos resulta adecuado para las plantaciones probadas y sus pruebas de bondad de ajuste y validación indican que se pierde poca precisión cuando se estiman las distribuciones diamétricas de las plantaciones probadas.

Los procedimientos de estimación de parámetros rápidos y fáciles parecen estar justificados. Esta justificación también ha sido corroborada por otras múltiples investigaciones. En comparaciones entre procedimientos de máxima verosimilitud y de porcentajes no se han encontrado diferencias significativas en el ajuste a estructuras diamétricas de plantaciones de Pinus taeda y se ha recomendado utilizar el segundo por su rapidez y facilidad para estimar parámetros (Zarnoch y Dell, 1985). Este último procedimiento se ha convertido en un método popular para estimar parámetros (Knowe et al., 1992; Newberry et al., 1993). Ueno y Osawa (1987), tampoco encontraron diferencias significativas entre las estructuras modeladas por la distribución Weibull de 2 y 3 parámetros ajustados por procedimientos de máxima verosimilitud y momentos para diámetros de Pinus densiflora, aunque por su facilidad y rapidez de estimación el segundo procedimiento de estimación fue recomendado.

\section{CONCLUSIONES}

Las estructuras diamétricas fueron ajustadas a la distribución Weibull por el procedimiento de momentos y sus parámetros predichos con atributos del rodal. El ajuste y predicción mostró buena precisión, demostrada por las pruebas de bondad de ajuste para los rodales utilizados en el ajuste y validación, respectivamente. Por esta razón, se recomiendan las ecuaciones obtenidas para modelar de manera preliminar las estructuras diamétricas de los bosques plantados de Pinus de la Sierra Madre Occidental de Durango, México. 


\section{RECONOCIMIENTOS}

Los autores de este reporte desean hacer patente su agradecimiento al Ing. José Guadalupe Barrios, Director Técnico de la UCODEFO No. 6 por las facilidades prestadas para la realización de este reporte. EI CONACYT y PAICYT apoyaron parcialmente los trabajos de este reporte a través de los proyectos de investigación 28536 B y CN 323-00, respectivamente. Los autores agradecen también a los árbitros quienes constructivamente enriquecieron el manuscrito final.

\section{REFERENCIAS}

Ayers G., D. y B. Jackson D. 1989. Simulation of purchase tract availability for timber harvesting annual planning. Forest Products Journal 39(5):59-63.

Bailey R., L. y T. Dell R. 1973. Quantifying diameter distributions with the Weibull function. Forest Science 19(2):97-104.

Clutter J., L.; J. Fortson C.; L. Pienaar V.; G. Brister H. y R. Bailey L. 1983. Timber Management: A Quantitative Approach. John Wiley \& Sons. Nueva York. pp:3-29.

Devore J., L. 1987. Probability and Statistics for Engineers and the Sciences. Brooks/Cole Publishing Company. California. 312 p.

Evans J., W.; R. Johnson A. y D. Green W. 1989. Two and three parameter Weibull goodness of fit tests. Research Paper Forest Products Laboratory. USDA Forest Service. No. FPL-RP-493. 27 p.

Gadow K., V. 1984. Fitting diameter distributions of even aged pine stands. Forstwissenschaftliches Centralblatt 103(6):360-374.
Graciano, L.J. 2001. Técnicas de evaluación dasométrica y ecológica de los bosques de coníferas bajo manejo de la Sierra Madre Occidental del centro sur de Durango, México. Tesis de Maestría. Facultad de Ciencias Forestales. UANL. Linares, N.L. México.

Gupta R.; K. Gebremedhin G. y M. Grigoriu D. 1992. Characterizing the strength of wood trust joints. Transactions of the ASAE 35(4):1285-1290.

Haan C., T. 1986. Statistical methods in hydrology. lowa State Press. 378 p.

Hafley W., L. y H. Schreuder T. 1977. Statistical distributions for fitting diameter and height data in even aged stands. Canadian Journal of Forest Research 7(3):481-487.

Hahn G., J. y S. Shapiro S. 1967. Statistical models in engineering. John Wiley \& Sons. Nueva York. 418 p.

Hokka H.; M. Piiroinen L. y T. Penttila. 1991. Estimation of basal area d.b.h. distribution using the Weibull function for drained pine and birch dominated peatland stands in north Finland. Folia Forestalia 781. 22 p.

Jayaraman K. y P. Rugmini. 1988. Diameter distributions for even aged teak. Indian Journal of Forestry 11(2):145-147.

Kilkki P.; M. Maltamo; R. Mykkanen y R. Paivinen. 1989. Use of the Weibull function in estimating the basal area d.b.h. distribution. Silva Fennica 23(4):311-318.

Knowe S., A.; T. Harrington B. y R. Shula G. 1992. Incorporating the effects of interspecific competition and vegetation management treatments in diameter distribution models for Douglas-fir saplings. Canadian Journal of Forest Research 22(9): 1255-1262. 
Kou W., Z. 1982. A study of the distribution of diameter of wood stems. Journal of Nanjing Technological College of Forest Products 1:51-65.

Laar A., V.; R. Mosandl y A. Van Laar. 1989. Diameter distributions in young oak stands. Allgemeine Forst und Jagdzeitung 160(9-10):189-194.

Li F., R. 1987. Study on diameter distribution and models predicting yields for natural Dahurian larch stands. Journal of North East Forestry University 15(4):8-16.

Liu X.; G. Wood R.; R. Woollons C. y A. White D. 1992. Stand table prediction with reverse Weibull and extreme value density functions: some theoretical considerations. Forest Ecology and Management 48(1-2):175 178.

Návar, J.; S. Corral y V. Dale. 2002. Fitting, estimating, and regressing the Weibull distribution parameters for pine and oak trees in mixed, unevenaged stands of Durango, Mexico. Submitted to Canadian Journal of Forest Research.

Návar, J. y J.C. Contreras. 2000. Ajuste de la distribución Weibull a las estructuras diamétricas de rodales irregulares de pino de Durango, México. Agrociencia 34:353-361.

Návar, J. y S. Corral. 2000. Modeling the Weibull distribution parameters of uneven-aged pine and oak diameter structures of Durango, Mexico. International Conference on Forest Ecosystem Restoration. 10-12 April 2000. Viena, Austria.

Newberry J., D.; J. Moore A.; L. Zhang J. y L. Zhang. 1993. Evaluation of simple quantile estimation functions for modelling forest diameter distribution in even-aged stands of interior Douglas-fir. Canadian Journal of Forest Research 23(11): 2376-2382.

Pierce C., B. 1976. The Weibull distribution and the determination of its parameters for application to timber strength data. Building Research Establishment, No. CP26/76. Reino Unido. 20 p.

Reynolds, M.R.; T.E. Burk y W. Huang. 1988. Goodness of fit tests and model selection procedures for diameter distribution models. Forest Science 34:373-399.

Seth S., K.; K. Satyamurthi R. y G. Negi S. 1975. A comparative study of some distribution functions as applied to diameter at breast height in even aged stands. Indian Forester 101(1):20-27.

Shiver B., D. 1998. Sample size and estimation methods for the Weibull distribution for unthinned slash pine plantation diameter distributions. Forest Science 34(3):809-814.

Svalov S.N. 1984. Using the generalized Gamma distribution for modeling the structure of stands. Lesnoi Zhurnal 2:10-13.

Tang Y. y R. Pearson G. 1992. Effect of juvenile wood and choice of parametric property distributions on reliability-based beam design. Wood and Fiber Science 24(2):216-224.

Torres R., J.M.; M. Acosta M. y O.S. Magaña T. 1992. Métodos para estimar los parámetros de la función Weibull y su potencial para ser predichos a través de atributos del rodal. Agrociencia: Rec. Nat. Ren. 2(2):60-76. 
Ueno Y. e Y. Osawa. 1987. The applicability of the Weibull and the expanded Weibull distributions. Journal of the Japanese Forestry Society 69(1):24-28.

Vanclay K., V. 1994. Modelling forest growth and yield: Applications to mixed tropical forests. CAB International. Wallingford, Oxon. Reino Unido. 312 p.

Wenger K., F. 1984. Forestry Handbook. Second Edition. Society of American Foresters. John Wiley \& Sons. Nueva York. 1335 p.
Zarnoch S., J. y T. Dell R. 1985. An evaluation of percentile and maximumlikelihood estimators of eibull parameters. Forest Science 31(1):260-268.

Zutter B., R.; R. Oderwald G.; R. Farrar M, Jr. y P. Murphy A. 1982. A program to estimate parameters of forms of the Weibull distribution using complete, censored and truncated data. Publication of School of Forestry and Wildlife Resources, Virginia Polytechnic Institute and State University. No. FWS pp:382. $\diamond$

1 Facultad de Ciencias Forestales. Universidad Autónoma de Nuevo León. Km 145 Carretera Nacional. Linares 67700 Nuevo León. México. Tel.: 821 24895. c.e.: jnavar@ccr.dsi.uanl.mx.

Manuscrito recibido el 6 de junio de 2001

Aceptado el 23 de agosto de 2001

Este documento se debe citar como:

Maldonado D., A y J. Návar Ch. 2002. Ajuste y predicción de la distribución Weibull a las estructuras diamétricas de plantaciones de pino de Durango, México. Madera y Bosques 8(1):61-72. 